\title{
Prevalence of Ocular Chlamydial Infections in South Florida
}

Darlene Miller, Jorge Mesa-Maestre, Edith Perez, Benjamin David Wilson and Eduardo C Alfonso

Bascom Palmer Eye Institute, University of Miami Miller School of Medicine, Miami, Florida, US

DOI: https://doi.org/10.17925/USOR.2016.09.02.102

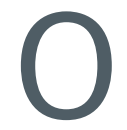

cular chlamydia in the US is a sexually transmitted disease. The true public health burden is unknown. We used a combination of culture and culture independent tests to document disease prevalence over a 28-year (1986-2014) period. Methods: Laboratory records of patient's samples $(n=3,112)$ submitted to rule out chlamydia were reviewed. Data were extracted for patient's demographics (age, sex), age and positivity rates for historical methods (1986-2004), direct fluorescent antibody (DFA; $n=1,774)$, culture ( $n=1,619$ ), nucleic acid amplification tests (NAAT; $n=274$ ) and enzyme immunoassay tests (EIA; $n=609)$ from historical data (1986-2014) versus the most recent 10 years, (DFA: $n=845)$ and culture $(n=523)(2005-2014)$. A multiplex polymerase chain reaction assay was used to determine species diversity among 128 random samples (1994-2014). Results: Chlamydial positivity rates ranged from $6.0 \%$ to $13.4 \%$, with an average of $10.2 \%$. The highest rates were documented for neonates (33.5\%) and the age group 15-29 (14.6\%, 24.5\%). Culture positivity was highest for nonculture tests (historical): NAAT (43.4\%), EIA (23.5\%), DFA (13.4\%) followed culture (8.5\%) and current DFA 7.6\% versus 3\% for culture. Mixed chlamydial infections were observed in 3.1\% of samples. A high prevalence and diversity of ocular chlamydia were evident in patients presenting with ocular surface infections in South Florida.

\section{Keywords}

Chlamydia, chlamydophila, positivity rate, direct fluorescent test, nucleic acid amplification test, enzyme immunoassay, prevalence.

Disclosure: Darlene Miller, Jorge Mesa-Maestre, Edith Perez, Benjamin David Wilson, and Eduardo C Alfonso have nothing to disclose in relation to this article. No funding was received in the publication of this article.

Compliance with Ethics: All procedures were followed in accordance with the responsible committee on human experimentation and with the Helsinki Declaration of 1975 and subsequent revisions, and informed consent was received from the patient involved in this study.

open Access: This article is published under the Creative Commons Attribution Noncommercial License, which permits any noncommercial use, distribution, adaptation, and reproduction provided the original author(s) and source are given appropriate credit.

Received: August 1, 2016

Accepted: September 14, 2016

Citation: US Ophthalmic Review, 2016;9(2):102-6

Corresponding Author: Darlene Miller, Bascom Palmer Eye Institute, 900 NW 17th Street, Miami Florida, 33136, US. E: dmiller@med.miami.edu
Few, if any studies are available on the prevalence and spectrum of ocular chlamydial disease in the US. Current estimates of ocular disease are predominantly anecdotal. The majority of disease is associated with urogenital transmission, with conservative estimated rates of one case for every 300 genital cases. According to Kalayoglu, 50-90\% of adults with ocular chlamydia have concomitant genital disease. ${ }^{1}$ Two thirds of neonates born to infected mothers will become colonized and one third of these will develop clinical evidence of neonatal inclusion conjunctivitis.

The true prevalence and public health burden, however, is unknown. None of the US sexually transmitted disease (STD) screening programs or clinics screen for ocular chlamydia. Nor do ophthalmologists and other ophthalmic healthcare providers routinely ask or screen for genital or extragenital infections.

The chlamydiae are non-mobile, gram negative, obligate, intracellular, energy parasites recovered from a variety of hosts, systemic, and ocular infections. Chlamydial species possess a unique and complex multiphasic developmental cycle, which requires living tissue for cultivation and growth.

A variety of ocular diseases are associated with chlamydia and chlamydia-like microorganisms worldwide. The order, Chlamydiales includes nine families, Chlamydiaceae, Clavichlamydiaceae, Criblamydiaceae, Piscicchlamydiacea, Parachlamydiaceae, Rhadochlamydiaceae, Simkaniaceae, Wadddliaceae and 'Candidatus' Parilichlamydiae more than 20 species. ${ }^{2}$ Currently, only members of two families (Chlamydiaceae and Parachlamydiaceae) and five species (C. trachomatis, C. pneumoniae, C. psittaci, Neochlamydia and Parachlamydia) have been associated with ocular infections. ${ }^{3-10}$

C. trachomatis causes the majority of ocular disease, including trachoma, ${ }^{2}$ neonatal and adult inclusion conjunctivitis, ${ }^{11,12}$ keratitis, ${ }^{1,13}$ uveitis, ${ }^{14}$ and lymphoma. ${ }^{15}$

C. pneumoniae is a major respiratory pathogen. Seroprevalence ranges from $40-80 \%$. Reports of ocular disease is rare. It has been recovered in patients in endemic trachoma area, ${ }^{16}$ patients with follicular conjunctivitis, ${ }^{17}$ and adults with keratoconjunctivitis. ${ }^{17}$

c. psittaci is endemic in pet and wild birds including parrots, pigeons, and parakeets. It causes psittacosis and or orthniosis, a systemic disease involving the conjunctiva. C. psittaci has been 
detected in endemic trachoma areas ${ }^{16}$ and in ocular adnexa lymphomas (OALS) tissues in patients from Italy and Asia. 14,18,19

\section{Family Parachlamydiaceae and family Rhadodochlamydiaceae}

These emerging chlamydial-like organisms are endosymbionts of free living amoeba (Acanthamoeba and or Hartmartnella) species. ${ }^{3,620}$ They can cause respiratory and ocular disease in humans., ${ }^{3,20}$ The spectrum of chlamydia species in ocular chlamydia is unknown.

Similar to genital disease, the diagnosis and detection of chlamydial species in ocular tissues can be difficult. ${ }^{21,22}$ Signs and symptoms often mimic other bacterial, viral infections, or allergic conjunctivitis. Chlamydia infections may present with only minor clinical signs and pose a significant therapeutic predicament for the ophthalmologist. 12,23

Complications following ocular chlamydia infections are varied. They may include and result in life-long and life-threatening sequelae, including chronic disease, corneal scarring, uveitis, asthma, and obstructive lung disease. ${ }^{123,24}$

\section{Methodology}

This was a laboratory-based surveillance to document prevalence and compare the diagnostic efficacy of three nonculture or antigen detection methods, direct fluorescent antibody (DFA), enzyme immunoassay (EIA), and nucleic acid amplification techniques (NAAT) relative to tissue culture to confirm the presence of chlamydia antigens in ocular tissue. Historical and current use data were collected from laboratory reports and entered into a SPSS database for comparison of performance characteristics and trends for two time periods (historical; 1986-2004, 18 years) and more recent (2005-2014, 10 years). Frequency and age specific rates were calculated per method and annually.

\section{Statistics}

Test comparisons were calculated for samples in which two or more diagnostic evaluations were performed. Prevalence data and cross tabulations on age and sex were evaluated using $2 \times 2$ chi square tables. McNemar's matched pair test $Z$ tests and or tests of proportions were used to determine significance and test performance characteristics (sensitivity, specificity, positive and negative predictive values). Data analysis was also supplemented using Excel.

\section{Patient population}

The patient population consisted of 3,112 consecutive patients $(1,640$ women and 1,472 men) evaluated at the Bascom Palmer Eye Institute Microbiology Laboratory to rule out ocular chlamydia from January 1986 to December 2014. Age ranged from 2 days to 98 years.

\section{Specimens}

Scrapings from ocular tissues, greater than $90 \%$ from the conjunctiva were collected with Dacron swabs or a platinum spatula after removable of obvious purulent discharge and placed within an inscribed circle on a glass slide for DFA. A second swab was collected and placed in chlamydia, EIA, polymerase chain reaction (PCR), or ligase chain reaction (LCR) transport media (2 ml) for culture, EIA, and/or nucleic acid amplification tests. Tissue biopsies from cornea or lids were placed in balanced salt solution in a sterile petri dish, sent to the microbiology laboratory for processing. Samples were collected and processed in accordance with manufacturer's instructions.

\section{Direct fluorescent antibody}

Scrapings were placed inside the well of the slide provided in the MicroTrak $^{\circledR}$ (Syva Inc, Palo Alto, CA, US). DFA kit and appropriately labeled with patient's name, source, and date of collection. Patient positive and negative control slides were fixed in acetone for 10 minutes. One drop (approximately $30 \mathrm{ul}$ ) of monoclonal antibody directed against the major outer membrane protein (MOMP) of chlamydia was added to each patient and or control well. Slides were incubated for 15 minutes at room temperature in a moist chamber. Slides were rinsed with sterile phosphate buffer for 10-15 seconds and air-dried. One drop of mounting fluid included in the kit was added to each patient and/or control well. The slides were screened with an epifluorescence microscope at a total magnification of 400x without oil. Positive slides were confirmed using a 100x oil immersion objective. Slides with less than five epithelial cell per high power field were rejected as inadequate. A positive result was scored as the presence of 10 or more apple green fluorescent round to oval elementary bodies (300 ul).

\section{Enzyme immunoassay}

Two-enzyme immunoassays were used to detect chlamydia in ocular tissue. The first of these, the Bartels PRIMA (Bartels [Intracel] Inc., Seattle, Washington) was a manual microwell system for detection of chlamydia antigen. Scrapings were collected and placed in the Bartels EIA transport medium or aliquots from cryopreserved or general chlamydia transport media (Bartels, Bartels, Seattle Washington) were processed according to manufacturer's suggestions.

The VIDAS chlamydia (BioMerieux, St. Louis, MO, US) is an enzyme-linked fluorescent immunoassay. All assay steps are performed and controlled by the instrument, but the principle is the same as the Bartel's PRIMA system. Scrapings were collected as above and placed into the VIDAS transport media. Samples were treated and processed in accordance with the manufacturer's instructions.

\section{Polymerase chain reaction}

The protocol for the detection of chlamydia by the polymerase chain reaction (PCR) was a manual protocol, designed in the laboratory in accordance with specifications of Bobo et al..$^{25}$ The 24 base pair primers (oligonucleotides) for the PCR (5'-A/CGT/TCG/CAG/GAA/GCC-3', 5'-AAC/TCA/AAA/CCC/TCT/CAT/TCT/ CAA-3') for the amplification of $C$. trachomatis were obtained from Genemed Biotechnologies, Inc (South San Francisco, CA, US). Thermocyclers used for the amplification of the product were purchased from the Perkin Elmer Corporation (Norwalk, CT, US) or Gene Machine (Atlanta, GA, US).

The reaction mixture was first heated to $94{ }^{\circ} \mathrm{C}$ for 1 minute, cooled to $55^{\circ} \mathrm{C}$ for 1 minute, followed by raising the temperature to $72{ }^{\circ} \mathrm{C}$ and incubating for 1 minute to extend the DNA chain. This procedure was repeated for a total of 32 cycles. A final 10-minute incubation at $72{ }^{\circ} \mathrm{C}$ was carried out for the ending cycle. A $2 \%$ agarose gel containing $0.5 \mu \mathrm{g} / \mathrm{ml}$ ethidium bromide was prepared, 10-20 $\mu \mathrm{l}$ of the amplified samples and or controls applied and subjected to electrophoresis for 2-4 hours. Detection of a band containing a 182 base pair product, with appropriate bands for positive and kit controls, complimented with no band for the negative control was scored as a positive result. 
Table 1: Historical methods-laboratory surveillance of ocular chlamydia (1986-2004)

\begin{tabular}{|c|c|c|c|c|c|c|c|c|c|c|}
\hline \multirow[t]{2}{*}{ Age Groups } & \multicolumn{3}{|l|}{ Patients } & \multicolumn{7}{|c|}{ Chlamydia Tests } \\
\hline & Females & Males & Total & DFA & Culture & NAAT-PCR & NAAT-LCX & EIA-(Bartels) & EIA (Vidas) & Total \\
\hline $0-1$ years & 156 & 169 & 325 & 256 & 267 & 26 & 64 & 96 & 52 & 761 \\
\hline 2-9 years & 61 & 78 & 139 & 108 & 113 & 7 & 8 & 24 & 25 & 285 \\
\hline 10-14 years & 66 & 47 & 113 & 97 & 90 & 2 & 8 & 25 & 20 & 242 \\
\hline 15-29 years & 238 & 243 & 481 & 420 & 344 & 15 & 51 & 72 & 64 & 966 \\
\hline 30-39 years & 164 & 149 & 313 & 274 & 239 & 3 & 18 & 38 & 43 & 615 \\
\hline 40-54 years & 172 & 157 & 329 & 279 & 245 & 12 & 28 & 28 & 39 & 631 \\
\hline 55-64 years & 99 & 67 & 166 & 139 & 127 & 4 & 16 & 16 & 16 & 318 \\
\hline$>65$ years & 94 & 42 & 136 & 112 & 106 & 1 & 6 & 19 & 21 & 265 \\
\hline NA & 45 & 54 & 99 & 89 & 88 & 3 & 2 & 5 & 6 & 193 \\
\hline Total & 1,095 & 1,006 & 2,101 & 1,774 & 1,619 & 73 & 201 & 323 & 286 & 4,276 \\
\hline
\end{tabular}

$D F A=$ direct fluorescent antigen; EIA = enzyme immunoassay; $L C X=$ ligase chain reactions; $N A=$ not applicable; $N A A T=$ nucleic acid amplifiction tests PCR $=$ polymerase chain reaction.

Table 2: Current methods-laboratory surveillance of ocular chlamydia (2005-2014)

\begin{tabular}{|c|c|c|c|c|c|c|}
\hline \multirow[t]{2}{*}{ Test period (s) } & \multicolumn{3}{|l|}{ Patients } & \multicolumn{3}{|l|}{ Tests } \\
\hline & Females & Males & Total & DFA & Culture & Total \\
\hline 2005-2009 & 263 & 216 & 479 & 392 & 279 & 671 \\
\hline 2010-2014 & 282 & 250 & 532 & 453 & 244 & 697 \\
\hline 2005-2014 & 545 & 466 & 1,011 & 845 & 523 & 1,368 \\
\hline percent & $53.9 \%$ & $46.1 \%$ & & $61.8 \%$ & $38.2 \%$ & \\
\hline
\end{tabular}

$D F A=$ direct fluorescent antigen.

\section{Ligase chain reaction}

The Abbott LCX LCR (Abbott Laboratories, Abbott Park, IL, US) is an automated procedure which employs oligonucleotide pairs to target gene sequences in the cryptic plasmid found in multiple copies in c. trachomatis or gene sequence in Neisseria gonorrhoeae. All tests were performed in accordance with the manufacturer' specifications. swabs with scrapings from conjunctiva and cornea or aliquots from cryopreserved samples were heated to $97^{\circ} \mathrm{C}$ for 15 minutes and cooled, specimens were expressed from the swabs and the swabs discarded. One hundred microliters of the remaining fluid was added to a $100 \mu \mathrm{l}$ tube LCR mixture. Amplification was carried out for 40 cycles for 1 second at $93{ }^{\circ} \mathrm{C}, 1$ second at $59{ }^{\circ} \mathrm{C}$ and 1 minute 10 seconds at $62{ }^{\circ} \mathrm{C}$. One hundred microliters of the amplified product was transferred to a reaction cell, which was placed in the analyzer for detection of chlamydia or N. gonorrhoeae.

\section{Culture}

Ocular samples for culture were received in chlamydia transport media, vortexed and $0.1 \mathrm{ml}$ of media inoculated into one light density and one moderate density cycloheximide-treated McCoy cells on glass coverslips in shell vials. Vials were incubated at $37{ }^{\circ} \mathrm{C}$ for $48-96$ hours and stained for chlamydia at 48 and 96 hours using the sylva Monoclonal antibody kit (Sylva, San Jose, CA, US) Specimens with greater than one inclusion or reticulate body were considered positive.

A Multiplex PCR using Qiagen Hot Start technique and species specific primers for C. pneumoniae and C. trachomatis were used to screen random samples collected between 1994 and 2014 to document the presence of nontrachomatis species and or mixed species among this group. Primers sequences included:

- Cpn 90 Madico F 5' GGTCTCAACCCCATCCGTGTCGg 3';

- Cpn 91 Madico R 5' TGCGgaAagCtGTATtTCtACAGTT 3';

- Ctr70 trachomatis F 5' GGCGTATTTGGGCATCCGAGTAACG $3^{\prime}$; and

- Ctr71 trachomatis R 5' TCAAATCCAGCGGGTATTAACCGCCT 3'.

The cycling parameters: one cycle at $95^{\circ} \mathrm{C}$ for 15 minutes, followed by 35 cycles with a denaturation step at $94{ }^{\circ} \mathrm{C}$ for 30 seconds, an annealing step at $62{ }^{\circ} \mathrm{C}$ for 30 seconds, and an extension step at $72{ }^{\circ} \mathrm{C}$ for 30 seconds, followed by one cycle of extension at $72{ }^{\circ} \mathrm{C}$ for 9 minutes. Amplified products were detected by electrophoresis.

\section{Gold standard}

Nonculture tests (DFA, Bartels-EIA, VIDAS-EIA, PCR and LCX) were compared with the 'gold' standard of a positive culture and an 'expanded gold standard' of positive culture and/or two positive nonculture tests. CDC defines positivity rates for chlamydia as the number of positive tests divided by the number of adequate tests performed.

\section{Results}

A total of 3,112 patients and 5,644 tests were evaluated over the 28 -year period. The ages ranged from two weeks to 98 years, with females constituting $52.7 \%(n=1,640)$ versus $47.3 \%(n=1,472)$ for males (see Tables 1 and 2).

Chlamydia antigens were documents in all age groups and both sexes by multiple test methods. Trends in historical and recent positivity chlamydia rates are displayed in Figure 1.

Chlamydia positive rates ranged from $5.8 \%$ to $13.4 \%$ with a median prevalence rate of $10.5 \%$. Overall prevalence (positivity) rates for ocular chlamydia steadily declined from the earliest test period, 1986-1989 (13.4\%) by twofold to 5.8\% ( $p=0.00014)$ for the last fiveyear test period (2010-2014). The culture positive rate was almost three times (2.7x) as high during the historical period 1986-2004, 8.5\% $(n=137 / 1619)$ compared to the last 10 years, 3.1\% $(n=523), 3.9 \%$ for test 
Figure 1: Trends in C. trachomatis conjunctivitis

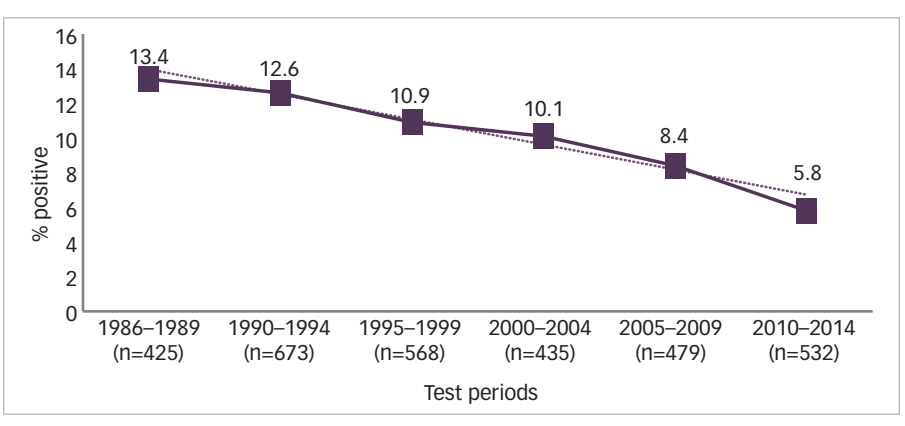

Figure 2: Age-specific chlamydia positivity rates 1986-2004

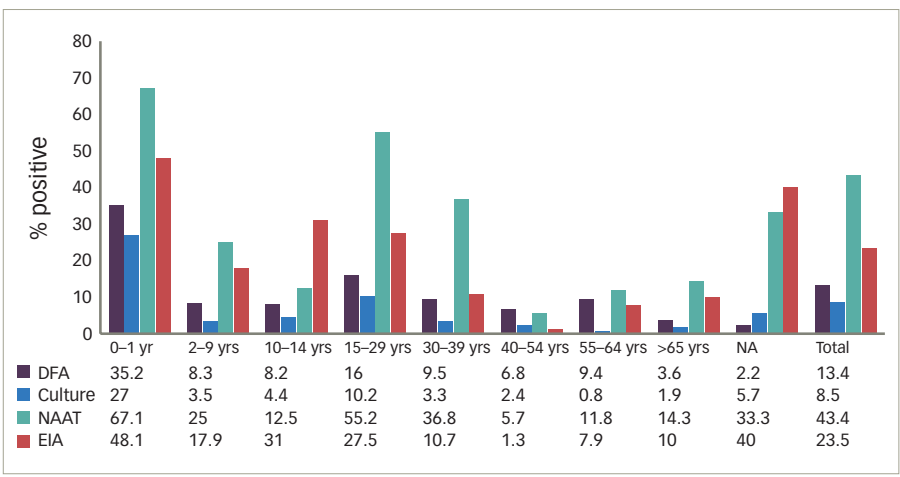

DFA = direct fluorescent antigen; EIA = enzyme immunoassay; $N A=$ not applicable; NAAT = nucleic acid amplifiction test; $y$ rs = years.

period 2005-2009 $(n=11 / 279)$ and $2.0 \%$ for the most recent test period 2010-2014 ( $n=5 / 244)$.

Figure 2 displays the historical age specific rate by test method. Age specific detection or positivity rates for chlamydia varied by tests methods. The highest rate of chlamydia was documented in age group 0-12 months for all tests and ranged from $27.3 \%$ (culture) to $73.9 \%$ (PCR). The second highest rate in five of the six tests was for age group 15-29 years (10.2\%-culture to $58.6 \%$-LCR). No significant differences between rates for age groups for culture (8.6\%) or the expanded gold standard (10.3\%). Age specific rates were also highest in males (12/120, 10\%, 24.5\%) and age group 15-29 (10/41, 24.5\%) for data collected for the last 30 months of period II (2005-2014).

Performance characteristics (sensitivity, specificity, PPV, and NPV) for the culture independent chlamydial tests versus culture as the gold standard of tissue culture are displayed in Table 3. The laboratory test with the highest values for the combination of sensitivity and negative predictive value to 'rule out' chlamydia relative to culture was Bartels EIA (93\% and 98\%, respectively). In descending order, results for the remaining tests were: DFA (85\% and 98\%), LCR (87\% and 92\%), PCR (77\% and 86\%), and VIDAS (69\% and $97 \%$ ). Compared to individuals and paired nonculture tests, culture was less than perfect.

C. pneumoniae $(n=13)$ was documented in $10.1 \%$ of the 128 screened samples versus $9.4 \%, n=12$ for $C$. trachomatis. Mixed chlamydia infections (C. trachomatis and C. pneumoniae) were found in four of the $13(30.8 \%)$ positive samples or $3.1 \%$ of tested samples (see Table 4).
Table 3: Performance characteristics—historical data

\begin{tabular}{|c|c|c|c|c|c|c|c|c|}
\hline NonCulture Tests & 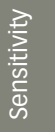 & $\begin{array}{l}95 \% \\
\mathrm{Cl}\end{array}$ & $\begin{array}{l}\frac{7}{0} \\
\frac{0}{0} \\
\frac{0}{0} \\
\mathbb{D} \\
\text { in }\end{array}$ & $\begin{array}{l}95 \% \\
\mathrm{Cl}\end{array}$ & PPV & $\begin{array}{l}95 \% \\
\mathrm{Cl}\end{array}$ & NPV & $\begin{array}{l}95 \% \\
\mathrm{Cl}\end{array}$ \\
\hline $\begin{array}{l}\text { Direct Fluorescent } \\
\text { Tests (DFA) }\end{array}$ & 85 & 76,92 & 93 & 91,95 & 55 & 47,64 & 98 & 97,99 \\
\hline Bartels ELISA & 93 & 83,98 & 81 & 76,86 & 54 & 44,64 & 98 & 95,99 \\
\hline Vidas ELISA & 69 & 47,87 & 95 & 92,97 & 59 & 39,78 & 97 & 94,99 \\
\hline NAAT-manual (PCR) & 77 & 56,91 & 77 & 63,88 & 64 & 45,81 & 86 & 72,95 \\
\hline $\begin{array}{l}\text { NAAT-automated } \\
\text { (LCR) }\end{array}$ & 87 & 76,94 & 83 & 75,89 & 73 & 62,83 & 92 & 86,97 \\
\hline Expanded Standard & 100 & 97,100 & 99 & 98,99 & 87 & 81,92 & 100 & 99,100 \\
\hline
\end{tabular}

$\mathrm{Cl}=$ confidence interval; $L C R=$ ligase chain reaction; $N P V=$ negative predictive value; $P C R=$ polymerase chain reaction; $P P V=$ positive predictive value .

\section{Discussion}

We provide a historical and current snapshot of the prevalence of chlamydia in the South Florida. This is the largest series reported to date. c. trachomatis was documented for all age groups by multiple test methods. Similar to genital rates, neonates, adolescents, and young adults were identified as high risk groups. Interestingly in this population, rates were higher for males than for females for all test periods. Females are more likely to be screened and perhaps treated than males. Currently, males are not routine targeted for screening in the US.

We are also the first group to document the presence of $C$. pneumoniae in ocular tissues of patients presenting with acute and or chronic chlamydial disease outside of an endemic trachoma area. C. pneumoniae in our series were detected with greater frequency than C. trachomatis. Coinfections occur in at least three or more patients.

As in genital disease nucleic acid amplification tests (NAATs) were the most sensitivity laboratory method for detecting chlamydia in ocular disease for both evaluation periods. A variety of authors have also documented the validity of nucleic acid tests for documentation of chlamydia in ocular tissue. Hammerschlag and colleagues evaluated AMPLICOR for the detection of $C$. trachomatis in ocular and nasopharyngeal specimens from 75 infants with conjunctivitis. They found the sensitivity, specificity and positive and negative predictive values of PCR compared with culture to be equivocal at $92.3,100,100$, and $98.4 \%$, respectively. ${ }^{25}$

Kowlaski and colleagues have also documented the efficacy of molecular methods to detect and confirm the presence of $C$. trachomatis in ocular samples. ${ }^{26,27}$

Currently, there are no approved NAATs tests for ocular chlamydia testing in the US. DFA and cultures remain the gold standards and current CDC recommendations. ${ }^{22}$

Although there was a steep decline from baseline $(13.4 \%, 1986-2004)$ to 6.0\% for the most recent years (2010-2014), overall rate (6.0\%) and rates for both males (7.6\%) and females (4.6\%) are equivocal or higher than the reported $1.7 \%$ for persons aged $14-39$ years (NHANES, 2007-2012) and the 4.7\% among sexually active females. 
Table 4: Spectrum of chlamydia species among patients with acute and chronic conjunctivitis, 1994-2014

\begin{tabular}{|l|l|l|l|}
\hline & $\begin{array}{l}\text { Number } \\
\text { positive } \\
\text { patients }\end{array}$ & $\begin{array}{l}\text { Number } \\
\text { patient } \\
\text { tests }\end{array}$ & \% Positive \\
\hline Chlamydophila pneumoniae & 13 & 128 & $10.1 \%$ \\
\hline Chlamydophila psittaci & 0 & 128 & 0 \\
\hline Chlamydia trachomatis & 12 & 128 & $9.4 \%$ \\
\hline Coinfections $^{\text {c }}$ & 4 & 128 & $3.1 \%$ \\
\hline
\end{tabular}

${ }^{a}$ Chlamydophila pneumoniae alone, 9/13, 69\% of total positives, 9/128=7.0\% for total tested; ${ }^{b}$ Chlamydia trachomatis alone, $8 / 12,67 \%$ of total positives, $8 / 128=6.2 \%$ for total tested; "Coinfections $-4 / 13$ or $30.7 \%$ of positive patients were infected two different Chlamydia species.

A total of 1,441,789 cases of genital chlamydia was reported in the US in 2014. The overall rate was 456.1 cases per 100,000 persons. Using the conservative rate of one case of ocular chlamydia for every 300 cases of genital disease, this would translate to 4,807 cases of ocular disease across the US or a rate of 1.52 per 100,000 persons. Miami-Dade is a highrisk area for chlamydia. The 2014 there was 10,814 reported cases for a rate of 406.1 per 100,000 people. The predicted rate for ocular chlamydia disease is 36 cases or a rate of 1.35 cases per 100,000 . This rate is likely incorrect and is likely to be higher. We documented 32 cases among 532 patients during 2010-2014.

Our data indicate a high prevalence of chlamydia in a patient population in a high risk area of the US and could signal an increasing public health burden.

\section{Limitations}

This was a retrospective review with the intrinsic problems associated with such studies. Data from laboratory and chart review may have been incomplete. Over the 28 year period, methods and technology and the environment in which a laboratory test was performed could have impacted the performance of screening tests. Patients evaluated for ocular chlamydia in this study may not have been representative of the general population of the larger group of patients with ocular conjunctivitis.

There may have been selection bias among patients referred or directed to our institute due to economic or recalcitrant therapeutic problems. In addition, patient samples were requested and submitted by physicians with varying clinical expertise in recognizing chlamydia. At least half of the patients were pretreated and this may have affected the documentation of chlamydia in these patients. $\square$
1. Kalayoglu MV, Ocular chlamydial infections: pathogenesis and emerging treatment strategies, Curr Drug Targets Infect Disord, 2002;2:85-91.

2. Dean D, Kandel RP, Adhikari HK, Hessel T, Multiple Chlamydiaceae species in trachoma: implications for disease pathogenesis and control, PLOS Med, 2008;5:e14

3. Corsaro D, Venditti D, Emerging chlamydial infections, Crit Rev Microbiol, 2004;30:75-106.

4. Corsaro D, Venditti D, Valassina $M$, New chlamydial lineages from freshwater samples, Microbiology, 2002;148(Pt 2):343-4.

5. Dean D, Bruno WJ, Wan R, et al., Predicting phenotype and emerging strains among Chlamydia trachomatis infections, Emerg Infect Dis, 2009:15:1385-94.

6. Everett KD, Bush RM, Andersen AA, Emended description of the order Chlamydiales, proposal of Parachlamydiaceae fam. nov. and simkaniaceae fam. nov., each containing one monotypic genus, revised taxonomy of the family Chlamydiaceae, including a new genus and five new species, and standards for the identification of organisms, Int J Syst Bacteriol, 1999;49:415-40.

7. Friedman MG, Dvoskin B, Kahane S, Infections with the chlamydialike microorganism Simkania negevensis, a possible emerging pathogen, Microbes Infect, 2003:5:1013-21.

8. Hammerschlag MR, Chlamydia and Chlamydiales: beyond Chlamydia trachomatis, Pediatr Infect Dis J, 2007;26:639-40.

9. Joseph SJ, Marti H, Didelot X, et al., Chlamydiaceae Genomics Reveals Interspecies Admixture and the Recent Evolution of Chlamydia abortus Infecting Lower Mammalian Species and Humans, Genome Biol Evol, 2015;7:3070-84.

10. Lamoth $F_{1}$ Jaton $K$ V VaudauX $B, G$ reub $G$, Parachlamydia and
Rhabdochlamydia: emerging agents of community-acquired respiratory infections in children, Clin Infect Dis, 2011;53:500-1.

11. Malathi J, Madhavan HN, Therese KL, Joseph PR, A hospital based study on the prevalence of conjunctivitis due to Chlamydia trachomatis, Indian J Med Res, 2003:117:71-5.

12. Mohile M, Deorari AK, Satpathy G, Sharma A, Singh M, Microbiological study of neonatal conjunctivitis with special reference to Chlamydia trachomatis, Indian J Ophthal, 2002;50:295-9.

13. Culbertson WW, Huang AJ, Mandelbaum SH, et al., Effective treatment of phlyctenular keratoconjunctivitis with ora tetracycline, Ophthalmology, 1993;100:1358-66.

14. Haller-Schober EM, El-Shabrawi Y, Chlamydial conjunctivitis (in adults), uveitis, and reactive arthritis, including SARA. Sexually acquired reactive arthritis, Best Pract Res CI OB, 2002:16:815-28.

15. Contini $C$, Seraceni $S$, Carradori $S$, et al., Identification of Chlamydia trachomatis in a patient with ocular lymphoma, Am J Hematol, 2009;84:597-9.

16. Lietman T, Brooks D, Moncada J, et al., Chronic follicular conjunctivitis associated with Chlamydia psittaci or Chlamydia pneumoniae, Clin Infect Dis, 1998;26:1335-40.

17. Krasny J, Hruba D, Netukova M, The role of Chlamydia pneumoniae in the etiology of keratoconjunctivitis sicca (KCS), Recent Pat Inflamm Allergy Drug Discov, 2014:8:216-22.

18. Ferreri AJ, Guidoboni M, Ponzoni M, et al., Evidence for an association between Chlamydia psittaci and ocular adnexal lymphomas, I Natl Cancer Inst, 2004;96:586-94.

19. Collina F, De Chiara A, De Renzo A, et al., Chlamydia psittaci in ocular adnexa MALT lymphoma: a possible role in
Iymphomagenesis and a different geographical distribution, Infect Agent Cancer, 2012;7:8.

20. Bush RM, Everett KD, Molecular evolution of the Chlamydiaceae Int J Syst Evol Microbiol, 2001;51(Pt 1):203-20.

21. CDC. FACT SHEET-2014 National Data on Chlamydia USA: 2015 2015. Report No.

22. Centers for Disease $C$, Prevention. Recommendations for the laboratory-based detection of Chlamydia trachomatis and Neisseria gonorrhoeae--2014. MMWR Recomm Rep. 2014;63 (RR-02): 1-19.

23. Sharma A, Satpathy G, Nayak N, et al., Ocular Chlamydia trachomatis infections in patients attending a tertiary eye care hospital in north India: a twelve year study, Indian J Med Res, 2012;136:1004-10

24. Lietman T, Dawson C, Osaki S, Ocular chlamydial infections, Int Ophthal Clin, 1998;38:125-35.

25. Hammerschlag MR, Roblin PM, Gelling M, et al., Use of polymerase chain reaction for the detection of Chlamydia trachomatis in ocular and nasopharyngeal specimens from infants with conjunctivitis, Pediatr Infect Dis I, 1997:16:293-7.

26. Kowalski RP, Karenchak LM, Raju LV Ismail N, The verification of nucleic acid amplification testing (Gen-Probe Aptima Assay) for chlamydia trachomatis from ocular samples, Ophthalmology 2015;122:244-7.

27. Kowalski RP, Thompson PP, Kinchington PR, Gordon YJ, Evaluation of the Smartcycler II system for real-time detection of viruses and Chlamydia from ocular specimens, Arch Ophthalmol, 2006;124:1135-9. 